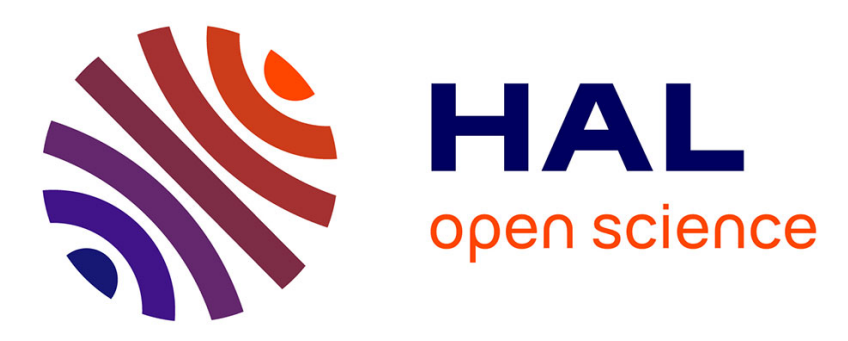

\title{
Miniature and cooled hyperspectral camera for outdoor surveillance applications in the mid-infrared
}

Armande Pola Fossi, Yann Ferrec, Nicolas Roux, Oscar D'almeida, Nicolas

\author{
Guerineau, Hervé Sauer
}

\section{To cite this version:}

Armande Pola Fossi, Yann Ferrec, Nicolas Roux, Oscar D'almeida, Nicolas Guerineau, et al.. Miniature and cooled hyperspectral camera for outdoor surveillance applications in the mid-infrared. Optics Letters, 2016, 41 (9), pp.1901-1904. 10.1364/OL.41.001901 . hal-01326809

\section{HAL Id: hal-01326809 \\ https://hal-iogs.archives-ouvertes.fr/hal-01326809}

Submitted on 30 May 2018

HAL is a multi-disciplinary open access archive for the deposit and dissemination of scientific research documents, whether they are published or not. The documents may come from teaching and research institutions in France or abroad, or from public or private research centers.
L'archive ouverte pluridisciplinaire HAL, est destinée au dépôt et à la diffusion de documents scientifiques de niveau recherche, publiés ou non, émanant des établissements d'enseignement et de recherche français ou étrangers, des laboratoires publics ou privés. 


\title{
Miniature and cooled hyperspectral camera for outdoor surveillance applications in the mid-infrared
}

\author{
Armande Pola Fossi, ${ }^{1, *}$ Yann Ferrec, ${ }^{1}$ Nicolas Roux, ${ }^{2}$ Oscar D'almeida, ${ }^{3}$ \\ Nicolas Guerineau, ${ }^{1}$ and Hervé Sauer ${ }^{4}$ \\ 'ONERA, Chemin de la Hunière, 91123 Palaiseau Cedex, France \\ ${ }^{2}$ SAGEM DS, 23 avenue Carnot, 91300 Massy, France \\ ${ }^{3}$ SAFRAN TECH, 1 rue Geneviève Aubé, CS 80112,78772, Magny Les Hameaux Cedex, France \\ ${ }^{4}$ Laboratoire Charles Fabry, Institut d'Optique Graduate School, CNRS, Université Paris-Saclay, 91127 Palaiseau Cedex, France \\ ${ }^{*}$ Corresponding author: armande.pola_fossi@onera.fr
}

Received 11 January 2016; accepted 14 March 2016; posted 23 March 2016 (Doc. ID 257185); published 19 April 2016

\begin{abstract}
We present the design and the realization of a compact and robust imaging spectrometer in the mid-infrared spectral range. This camera combines a small static Fourier transform birefringent interferometer and a cooled miniaturized infrared camera in order to build a robust and compact instrument that can be embedded in an unmanned aerial vehicle for hyperspectral imaging applications. This instrument has been tested during a gas detection measurement campaign. First results are presented. (C) 2016 Optical Society of America
\end{abstract}

OCIS codes: (110.3080) Infrared imaging; (110.4234) Multispectral and hyperspectral imaging; (110.0110) Imaging systems; (260.1440) Birefringence; (120.4640) Optical instruments.

http://dx.doi.org/10.1364/OL.41.001901

Recent developments in unmanned aerial vehicles (UAVs) have increased the demand for more compact and quicker plug-andplay optical instruments for outdoor measurements. The design of these UAV-embedded cameras is governed by a trade-off between compactness and performance. In the case of hyperspectral imaging sensors, which gather spatial and spectral information of a scene, this trade-off can be made between the size of the whole system and the finesse. We define the finesse of a hyperspectral camera as the ratio between the wavenumber and the spectral resolution. For compact systems, its value is typically around hundreds of bands [1-3]. The spectral sampling can be obtained by means of dispersive elements (prisms or gratings), by filters (Bragg filters or acousto-optic tunable filters, for example), or by temporal or static Fourier transform spectrometer (FTS). The static Fourier transform acquisition mode presents the advantage of having more flux and of being robust, as it has no moving components.

Most of the imaging spectrometers based on static Fourier transform use a lateral shearing interferometer, which is a twowave interferometer that splits an incident wavefront into two replicas, laterally displaced with respect to each other. The fringes of this type of interferometer are localized at infinity, and are nearly linear and equidistant in the paraxial regime, when imaged on the focal plane. The imaging system superimposes the fringes with the scene, which leads to an intensity-modulated image. The device is then installed on a scanning system, which enables the construction of the interferogram of each point of the scene by its movement. The principle is presented in Fig. 1. Usually a Michelson [4,5] or a Sagnac [6,7] interferometer is used, but it is quite bulky due to the use of a beam splitter. In order to have a compact system, we have studied the possibility of a volume reduction of the interferometer and of the imaging device.

Regarding the miniaturization of the interferometer, we have studied polarization interferometers that use the double refraction in uniaxial plates [8] to split a beam into two orthogonally polarized beams, said ordinary and extraordinary polarized. These interferometers are composed of a combination of uniaxial plates put between crossed polarizer and analyzer, with their axes oriented at $45^{\circ}$ to the principal sections (plane defined by the optical axis of the uniaxial crystal and the normal to the dioptre) of the plates in order to maximize the fringes contrast. The combination of plates is usually composed of two plates, the second plate being an optical path difference (OPD) compensator for a given incidence angle, in order to

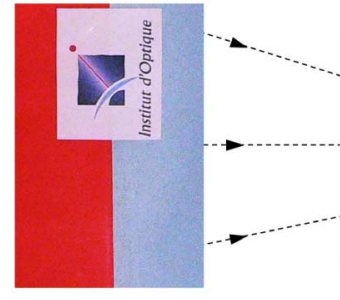

Scene at infinity

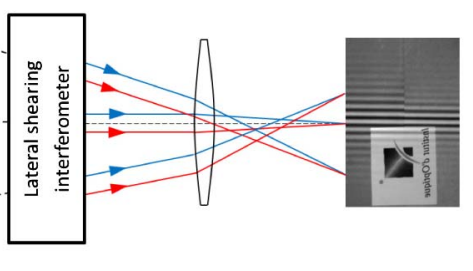

Imaging lens
Modulated scene
Fig. 1. Static imaging FTS based on a lateral shearing interferometer; each incident ray is split into two emerging rays (blue and red) separated by a constant translation. The scanning system is not shown. 


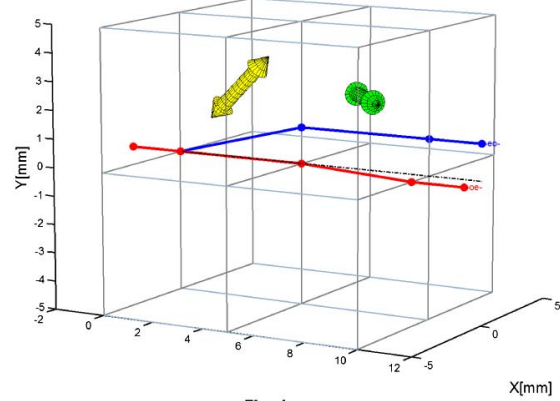

$\mathrm{Z}[\mathrm{mm}]$

Fig. 2. Savart plates, the uniaxial material optical axes (shown as $3 \mathrm{D}$ arrows) are orientated at $45^{\circ}$ in the $\mathrm{YZ}$ and $\mathrm{XZ}$ planes, respectively, $\mathrm{Z}$ being the instrument main axis, the two optical paths are also presented in blue and red colors.

reach zero OPD. In the literature, we have found four types of birefringent lateral shearing interferometers made of a combination of uniaxial plates of the same material: the Savart plates, illustrated in Fig. 2; the modified Savart plates, where an additionnal achromatic half-wave plate is inserted between the plates of which optical axes are oriented at $45^{\circ}$ and lie in the $X Y$ plane; the double-Wollaston (DW) made of four prisms $[9,10]$, but which can be interpreted as two plates with one inserted inside the second; and finally, a combination patented by Phua and Lim, where the optical axis of the first plate is oriented at $45^{\circ}$ with respect to the $Z$ axis and lies in the $Y Z$ plane, the optical axis of the second plate being oriented at $90^{\circ}$ to the $Z$ axis and lying in the $X Z$ plane [11].

Such static birefringent interferometers have been mainly developed in the visible range [12-14], but to the best of our knowledge, there is no system in the infrared spectral range. We propose here to detail the realization of a Savart-based spectral imager in the mid-infrared. In fact, the Savart interferometer was preferred because the modified Savart and the DW interferometers presented more manufacturing constraints, either for the achromatic half-wave retarder or for the four prisms with the same angle. No fundamental reason made us choose between the Savart interferometer and the design proposed by Phua and Lim, apart from the fact that the Savart interferometer is more widespread in the literature.

In a FTS, the finesse $(\mathcal{F})$ is defined as

$$
\mathcal{F}=2 \cdot \sigma_{\text {max }} \cdot \delta_{\text {max }}
$$

with $\sigma_{\max }$ being the maximum wavenumber of the spectral range and $\delta_{\max }$ the maximum achievable OPD. Thus designing a compact system while keeping a relevant finesse of $\sim 200$, implies increasing the maximum OPD of the interferometer as much as possible. Equation (2), where $n_{e}, n_{o}$ are, respectively, the extraordinary and the ordinary indices and $\omega$, the angle between the principal section and the incidence plane, gives the OPD of the Savart interferometer [9]. One can see that the OPD increases with the common thickness $(e)$ of the plates, the field angle $\left(\theta_{i}\right)$ and the birefringence $\left(\Delta n=n_{e}-n_{o}\right)$.

$$
\begin{aligned}
\frac{\delta}{e}= & \frac{n_{o}^{2}-n_{e}^{2}}{n_{o}^{2}+n_{e}^{2}}(\cos (\omega)+\sin (\omega)) \cdot \sin \left(\theta_{i}\right)+\frac{n_{o} \sqrt{2}}{2 n_{e}} \\
& \cdot \frac{n_{o}^{2}-n_{e}^{2}}{\left(n_{o}^{2}+n_{e}^{2}\right)^{3 / 2}} \cdot\left(\cos (\omega)^{2}-\sin (\omega)^{2}\right) \cdot \sin \left(\theta_{i}\right)^{2} .
\end{aligned}
$$

We started the optical design by looking for highly birefringent materials transparent in our spectral range and of which the manufacturing process is totally mastered. This led us to $\mathrm{TeO}_{2}$, which has birefringence of 0.14 at $4.1 \mu \mathrm{m}$. To reach a wide field-of-view (FOV) while keeping the system compact, we chose to use a prototype camera named "SOIE" [15], which is an ultracompact wide field $\mathrm{F} / 4$ cryogenic camera whose lens is fully integrated in the cooled-detector Dewar. This camera, inspired by landscape lens design [16], is composed of a meniscus lens with its concave surface toward the object, a cold aperture stop, and $\mathrm{HgCdTe}$ focal plane array (FPA). The position of the aperture stop, the lens bending, and the lens glass (silicon) have been defined to minimize astigmatism, spherical, coma aberrations, axial, and lateral chromatism. An optical filter was also inserted to limit the spectral range between $3.7 \mu \mathrm{m}$ and $4.8 \mu \mathrm{m}$, but it could be changed if a wider spectral domain is needed. The "SOIE" wide total FOV of $60^{\circ} \times 48^{\circ}$ led to $15 \mathrm{~mm}$ for the $2 e$ total thickness of the Savart plate interferometer to reach the aimed finesse. In order to improve the spectral resolution, the classical Savart polariscope has been modified by introducing an asymmetry in the geometry. Indeed, instead of two plates of equal thickness, the thickness of the second one was reduced to shift the zero-OPD to an incidence angle different from the normal incidence. This asymmetry increases the maximum achievable OPD from $250 \mu \mathrm{m}$ to $420 \mu \mathrm{m}$ and thus improves the theoretical spectral resolution to $11.9 \mathrm{~cm}^{-1}(\sim 19 \mathrm{~nm}$ at $\lambda=4 \mu \mathrm{m})$. The layout of the system is presented in Fig. 3 .

For this first prototype, called SIBI for "Spectro-Imageur Biréfringent Infrarouge," the design of the SOIE camera has not been modified and we have a $8 \mathrm{~mm}$ distance between the Dewar window and the cold stop. This leads to an interferometer with a large aperture of $40 \mathrm{~mm}$ for the plates. $\mathrm{TeO}_{2}$ crystal plates have been bonded together by optical contact and antireflective coated by Altechna. The high image quality polarizer with a transmission efficiency of $93 \%$ was bought from Specac. The overall weight of the instrument is $2.7 \mathrm{~kg}$ and its volume is $12 \mathrm{~cm} \times 12 \mathrm{~cm} \times 20 \mathrm{~cm}$.

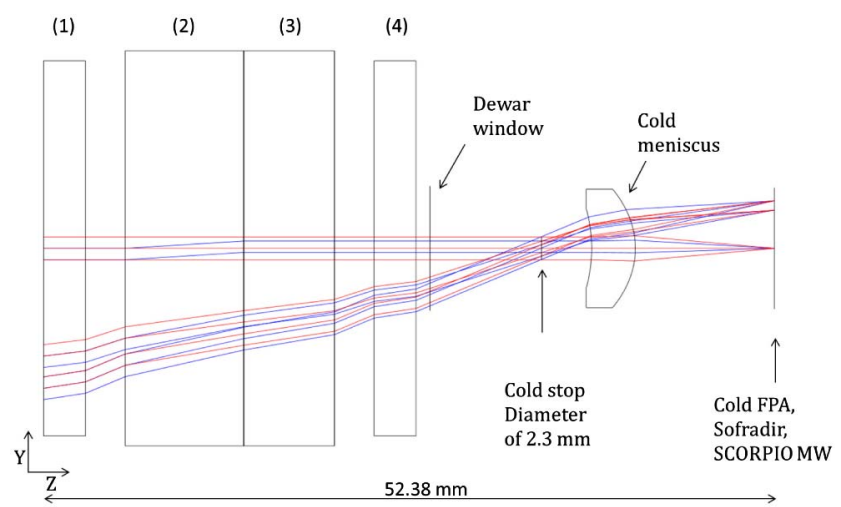

Fig. 3. Layout of the system. (1) and (4) are, respectively, the polarizer and the analyzer. (2) and (3) are the $\mathrm{TeO}_{2}$ plates of the dissymmetrical Savart. The interferometer is put at $1 \mathrm{~mm}$ in front of the Dewar window. Rays in red represent the OE optical path and rays in blue the EO optical path (OE and EO denote the two optical paths in the Savart interferometer; denomination related to the polarization state of the beam while propagating through the system, extraordinary or ordinary). 


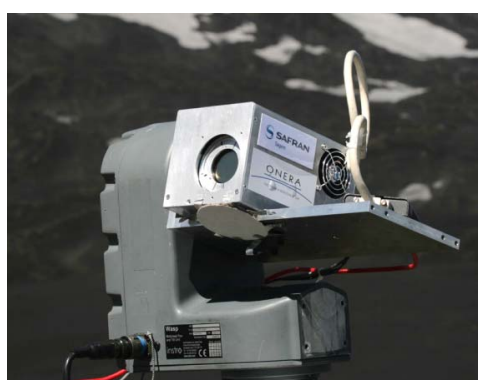

Fig. 4. SIBI $(12 \mathrm{~cm} \times 12 \mathrm{~cm} \times 20 \mathrm{~cm}$ cube with logos $)$ installed on a rotation stage for gas detection in Etna plume.

At the end of June 2015, SIBI has been involved in a gas detection measurement campaign in the plume of the Etna volcano. The goal of this campaign, which was led by the LPC2E (Laboratoire de Physique et Chimie de l'Environnement et de l'Espace) and the LATMOS (Laboratoire Atmosphères, Milieux, Observations Spatiales), was to compare the detection capability of different instruments [17]. Here the scanning was done by a rotating stage, as illustrated by Fig. 4 .

The calibration of the instrument was made on the field just before the acquisition of a series of measurements. The image stack we processed was acquired in the morning, and the ambient temperature was $9^{\circ} \mathrm{C}$. This sequence contained 1500 measurement images at a given integration time and calibration black body images at different temperatures and integration times. The image processing was done in four stages: the nonuniformity correction (NUC), the geometrical distortion correction, the image registration, and finally, the application of a Fourier transform algorithm to recover the spectrum.

Nonuniformities are due to the disparity of output voltage of the pixels in response to a uniform illumination. To correct this disparity, a uniform black body image at a temperature of $5^{\circ} \mathrm{C}$, called the background image, was subtracted from the images of the sequence. An image with a zero integration time, called noise image, was used to find bad pixels location by thresholding. Once bad pixels were located, their values in each image of the sequence were replaced by the mean value of the neighboring good pixels. Afterward, the pixel gain was corrected. For this, the background image was subtracted from a black body image $\left(\operatorname{im}_{B B}\right)$ at $15^{\circ} \mathrm{C}$, and the result was corrected from bad pixels. Interference fringes, which modulate the response of detector pixels in $\mathrm{im}_{B B}$, were suppressed by applying a mask in the Fourier space to set the spatial frequencies due to the fringes to zero. The pixel gain was defined as the inverse of the black body image without the fringes obtained previously.

For the geometric distortion correction, we proceeded in two steps. First, we corrected the optical distortion by using a pinhole camera model for a third order radial distortion and a first order tangential distortion $[18,19]$. This was done in the laboratory, installing SIBI on a two-axes rotation stage and putting it in front of a collimated black body source. We have used a least square method to recover the coefficients of the model for which the modeled distorted coordinates of the spot centroid best fit the measured ones. To evaluate the fitting quality of the model, we used the Euclidean distance in pixels

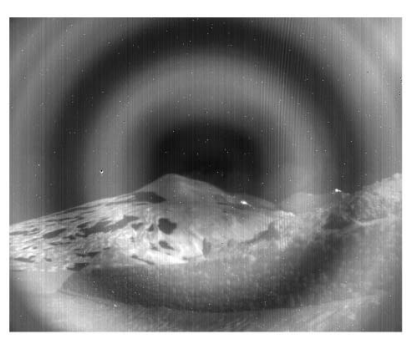

(a)

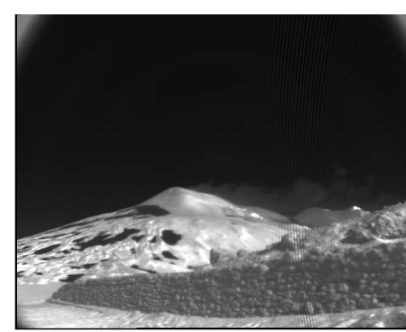

(b)
Fig. 5. (a) Example of raw image. One can see the narcissus effect of the detector, the bad pixels, and stripes. (b) Corrected image from nonuniformities and geometrical distortions.

between the estimated position and the measured position of the spot centroid. We found a mean error of 0.17 pixels in the full FOV. Second, we corrected the deformations introduced by the scanning system. Indeed, the homography transformation introduced by the camera panning was transformed into a translation transformation before applying a correction algorithm for motion estimation. This is done by using cylindrical panoramic projection [20]. Let us specify that to transform a distorted image into an undistorted one, regularly sampled on a cylindrical grid, a relationship was found between the distorted coordinates and the coordinates of the centroid on the theoretical cylindrical FPA, so that only one interpolation was performed to correct the image. Figure 5 shows an example of the correction of a raw image.

The following stage of the image processing was the registration of the stack of images now related by a translation transformation. For this, a reference image was selected and the translation vector $\vec{u}$ between this image and the other images was estimated using a correlation algorithm based on Eq. (3):

$$
\vec{u}=\underset{x, y}{\arg \max }\left(\mathrm{FT}^{-1}\left[\frac{\widetilde{I_{1}} \cdot{\widetilde{I_{2}}}^{*}}{\left|\widetilde{I}_{1} \cdot{\widetilde{I_{2}}}^{*}\right|}\right](x, y)\right),
$$

where $\mathrm{FT}^{-1}$ is the inverse Fourier transform, $\widetilde{I}$ is the Fourier transform of image $I$, and $I^{*}$ the Hermitian conjugate of $I$. Next, the parameters of the motion model which transforms the coordinates of the reference image into the coordinates of the image to be registered were fitted by a first order polynomial and used for the registration of the 875 images for which the FOV overlaps. The same motion model has been applied to the OPD image to construct the interferogram.

Finally, we applied a Fourier transform algorithm to the interferograms to recover the spectra of all pixels. These latter were divided by the spectral sensitivity of SIBI, and we added to them the Planck law for a blackbody at $5^{\circ} \mathrm{C}$, to compensate for the background subtraction operated on the raw images. We thus obtained spectra calibrated in radiance. Preliminary results are presented in Fig. 6. The experimental spectral resolution here is $13 \mathrm{~cm}^{-1}(\sim 21 \mathrm{~nm}$ at $\lambda=4 \mu \mathrm{m})$.

In conclusion, a new compact and robust hyperspectral camera, SIBI, which operates in the midwavelength infrared spectral range, has been designed and built. This hyperspectral camera has been tested in real operating conditions, and the 


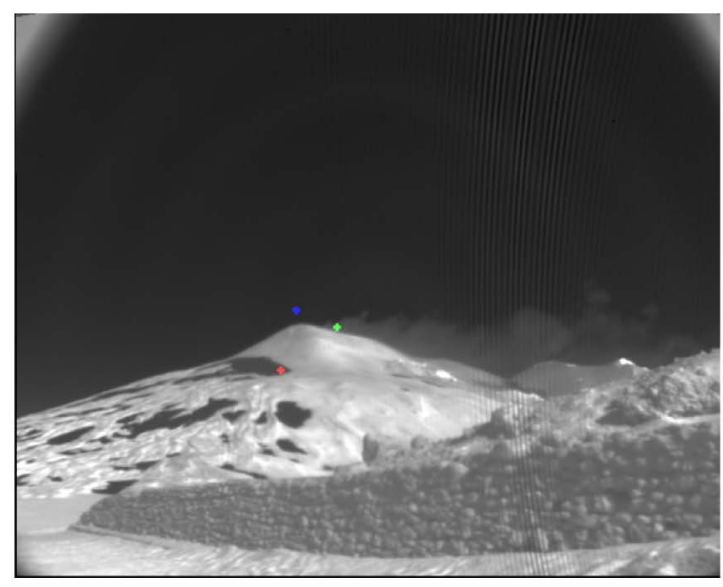

(a)

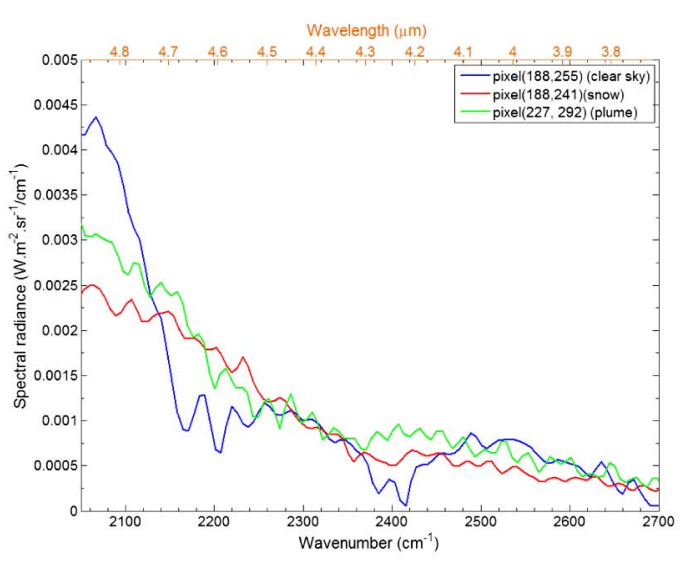

(b)

Fig. 6. (a) Location of an example of the points for which the spectra have been calculated: point in the sky (blue), in the plume (green) and in the snow (red). Note that snow appears black in this thermal-IR picture as it is colder than the rocks beside. (b) Obtained spectra, preliminary results.

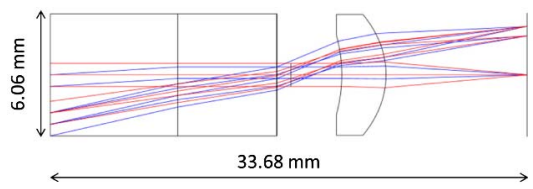

Fig. 7. Optical scheme of a more compact imaging spectrometer (same scale as Fig. 3). Polarizer and analyzer are directly deposited on the external faces of the $\mathrm{TeO}_{2}$ uniaxial plates and the Dewar window has been brought closer to the cold stop.

first results are encouraging. In future work, we will improve our image processing chain, as some artifacts have been noticed in the final images. The optomechanics will also be redesigned for a UAV-embedded measurement campaign. The optical design itself will also be reconsidered. Indeed, in the present configuration, there is a useless distance of $8 \mathrm{~mm}$ between the cold stop and the Dewar window. Removing this gap and the optical filter as well as depositing the polarizer and the analyzer directly on the surface of the Savart plates, which is the main challenge here, will reduce the aperture of the plates by almost three times, and thus, decrease the whole size of the system. Figure 7 presents the layout of this new system at the same scale as the layout of Fig. 3.

Funding. Labex Voltaire (ANR-10-LABX-100-01); French National Program of Chemistry, CNRS-INSU (LEFE-CHAT).

Acknowledgment. The authors thank Christophe Coudrain, Guillaume Druart, Mathieu Chambon, Roland Domel and SOFRADIR for the help they brought to us during this work. We are also grateful to ALTECHNA teams for their availability during the manufacturing of the interferometer. We also thank Labex Voltaire (ANR-10-LABX-100-01) from the ANR agency, and LEFE-CHAT, French National Program of Chemistry from CNRS-INSU, for funding.

\section{REFERENCES}

1. G. Bareth, H. Aasen, J. Bendig, M. L. Gnyp, A. Bolten, A. Jung, R. Michels, and J. Soukkamäki, Photogrammetrie-FernerkundungGeoinformation 2015, 69 (2015).

2. S. L. Hill and P. Clemens, Proc. SPIE 9482, 94821E (2015).

3. N. Tack, A. Lambrechts, P. Soussan, and L. Haspeslagh, Proc. SPIE 8266, 82660Q (2012).

4. C. Coudrain, S. Bernhardt, M. Caes, R. Domel, Y. Ferrec, R. Gouyon, D. Henry, M. Jacquart, A. Kattnig, P. Perrault, L. Poutier, L. RoussetRouvière, M. Tauvy, S. Thétas, and J. Primot, Opt. Express 23, 16164 (2015).

5. D. Bergstrom, I. Renhorn, T. Svensson, R. Persson, T. Hallberg, R. Lindell, and G. Boreman, Proc SPIE 7660, 76602F (2010).

6. D. Cabib, R. Buckwald, M. Lavi, and A. Gil, "New airborne pushbroom spectral imager for the 3-5 and 7-12 $\mu \mathrm{m}$ wavelength ranges," in Conference Proceedings of the XXXI Symposium of the Optical Society of India, Dehradun, India (2005).

7. P. G. Lucey, M. Wood, S. T. Crites, and J. Akagi, Proc. SPIE 8390 $83900 \mathrm{Q}(2012)$.

8. M. Born and E. Wolf, Principles of Optics (Cambridge University, 1999).

9. M. Françon and S. Mallick, Polarization Interferometers (Wiley-Interscience, 1971).

10. A. Harvey and D. Fletcher-Holmes, "Imaging apparatus," patent WO20045870 A1 (April 15, 2004).

11. P. Phua and B. Lim, "Hyperspectral imaging device," WO patent PCT/ SG2011/000,022 (January 17, 2011).

12. G. Zhan, K. Oka, T. Ishigaki, and N. Baba, Appl. Opt. 41, 734 (2002).

13. J. Courtial, B. Patterson, A. Harvey, W. Sibbett, and M. Padgett, Appl. Opt. 35, 6698 (1996).

14. C. Zhang, X. Bin, and B. Zhao, Proc. SPIE 4087, 957 (2000).

15. G. Druart, F. de la Barriere, N. Guérineau, J. Deschamps, M. Fendler, N. Lhermet, J. Rulliere, S. Magli, Y. Reibel, and J. B. Moullec, Proc. SPIE 8012, 801228 (2011).

16. M. Bass, Handbook of Optics (McGraw-Hill, 1994).

17. S. Payan, N. Huret, V. Catoire, S. Langlois, G. Salerno, T. Roberts, D. Rodriguez, A. Pola Fossi, A. La Spina, T. Caltabiano, S. Chevrier, Y. Ferrec, and M. Burton, in AGU Fall Meeting (AGU, 2015), paper V23B-3098.

18. D. C. Brown, Photogramm. Eng. Remote Sens. 37, 855 (1971).

19. A. E. Conrady, Mon. Not. R. Astron. Soc. 79, 384 (1919).

20. D. Salomon, Transformations and Projections in Computer Graphics (Springer, 2007). 